\title{
Opérations des fractures vertébrales ostéoporo- tiques: le Medical Board préconise la retenue
}

\author{
Les fractures vertébrales dues à l'ostéoporose peuvent être stabilisées avec des injec- \\ tions de ciment (vertébroplastie ou kyphoplastie). Toutefois, comparé au traitement \\ conventionnel, les effets à moyen terme de l'injection de ciment sont sujets à cau- \\ tion. Le Swiss Medical Board conseille de vérifier minutieusement, au moyen d'études \\ prospectives, chez quels patients l'opération peut s'avérer efficace.
}

Swiss Medical Board* * Organisation et composition
sous www.medical-board.ch/
index.php?id= $818 \& \mathrm{~L}=1$
Correspondance:

Susanna Marti Calmell Secrétariat Swiss Medical Board Obstgartenstrasse 21

$\mathrm{CH}-8090$ Zurich

Tél. 0432592479

Info[at]medical-board

www.medical-board.ch
L'ostéoporose (ou maladie des os fragiles) est une maladie du squelette caractérisée par un déséquilibre entre la formation normale des os et la résorption osseuse conduisant à une augmentation du risque de fractures vertébrales. En Suisse, le nombre total de fractures vertébrales ostéoporotiques diagnostiquées peut être estimé à environ 19000 par an.

Pour traiter les fractures vertébrales ostéoporotiques, deux types de procédures existent: les procédures conservatrices et les procédures chirurgicales. Les principales procédures chirurgicales sont la vertébroplastie et la kyphoplastie, qui consistent à injecter un ciment osseux dans le corps vertébral au moyen d'une aiguille creuse afin de stabiliser les vertèbres effondrées.

L'étude du Medical Board porte sur la question de savoir si les procédures chirurgicales (vertébroplastie ou kyphoplastie) sont plus efficaces que les procédures conservatrices pour réduire les symptômes de la maladie. Les auteurs ont également tenu compte des effets potentiellement négatifs et du rapport coût-efficacité.

D'après la littérature existante, les deux interventions chirurgicales précitées permettraient effectivement d'améliorer les symptômes d'une fracture vertébrale à court terme, mais aucune différence cliniquement significative ne peut cependant être observée, ni du point de vue de l'intensité de la douleur ni de l'amélioration fonctionnelle, entre le traitement chirurgical et le traitement conservateur après une période de 6 mois.

Les spécialistes consultés ont convenu que la vertébroplastie et la kyphoplastie sont efficaces pour le sous-groupe de patients qui ne montrent pas d'amélioration significative de la douleur et de la fonctionnalité malgré plusieurs semaines de traitement conservateur approprié.

Différentes études indiquent par ailleurs que les deux procédures chirurgicales étudiées conduisent à une qualité de vie en moyenne légèrement meilleure. Le rapport coût-efficacité s'élève à 108000 francs par QALY lorsque la procédure est menée essentiellement en stationnaire, ce qui signifie que la procédure opératoire est efficace, mais exige en même temps des dé- penses supplémentaires. Le rapport coût-efficacité se situe dans une zone dans laquelle il convient d'examiner avec attention si l'intervention doit être facturée à la charge de l'assurance-maladie sociale. Le rapport coût-efficacité est en revanche plus favorable (59 000 francs par QALY) lorsque la procédure est effectuée principalement en ambulatoire.

Les conclusions de cette étude conduisent aux recommandations suivantes:

- La question de savoir quel groupe de patients est susceptible de voir rapidement ses douleurs diminuer, et ainsi retrouver son indépendance, grâce à une vertébroplastie ou à une kyphoplastie réalisée durant la phase précoce suivant une fracture d'un corps vertébral doit être résolue par des études prospectives appropriées.

- Les obligations liées à la certification et à la tenue de registres qu'exige l'ordonnance sur les prestations de l'assurance des soins (OPAS) pour la kyphoplastie doivent être également déclarées obligatoires pour la vertébroplastie.

- La réalisation d'une vertébroplastie ou d'une kyphoplastie pour traiter les fractures vertébrales ostéoporotiques en dehors d'une étude contrôlée devrait être effectuée sur la base d'une indication multidisciplinaire et ce uniquement pour les patients souffrant de douleurs persistantes.

\section{Swiss Medical Board}

La mission du Swiss Medical Board consiste à analyser des processus diagnostiques et des interventions thérapeutiques du point de vue médical, économique, éthique et juridique. II s'agit notamment d'évaluer le rapport entre les coûts et l'efficacité des prestations médicales. Le Swiss Medical Board formule des recommandations à l'attention des décisionnaires politiques et des fournisseurs de prestations. Le Swiss Medical Board est un organisme indépendant de l'administration, des fournisseurs de prestations et de l'industrie, qui comprend un conseil d'experts ainsi qu'un bureau. L'organisation de soutien du Swiss Medical Board comprend la CDS, la FMH et l'ASSM.

Informations complémentaires: www.medical-board.ch 